\title{
Editors' introduction to the special issue: papers from LREC 2014
}

\author{
Nancy Ide ${ }^{1} \cdot$ Nicoletta Calzolari $^{2}$
}

Published online: 27 May 2016

(C) Springer Science+Business Media Dordrecht 2016

This special issue of Language Resources and Evaluation includes a selection of extended papers from the Ninth Language Resources and Evaluation Conference, held in Reykjavik, Iceland, in May 2014. The conference drew a record number of participants, reflecting the steadily increasing interest and activity in the field of language resource creation, enhancement, management, and evaluation since the first LREC was held in 1998. The selection of articles for this special issue was made on the basis of recommendations from LREC reviewers, who were asked to indicate suitability for publication of an extended version in $L R E$ for each paper they reviewed.

The range of resources represented by the papers selected for this special issue spans both familiar resource types as well as newer phenomena such as YouTube audio (Rodriguez-Fuentes et al.) and richly annotated multi-modal data (Malisz et al.). Methodologically, the papers demonstrate the steady advancement of techniques for resource development and evaluation. For example, Rosén et al. describe a method for mutual, iterative enhancement of treebanks and the lexicon; and Laparra et al. describe the use of graph-based disambiguation algorithms and corpus alignment to automatically establish mappings among semantic resources. Xia et al. describe the expansion of ODIN, a database containing Interlinear Glossed Text (IGT) for over a thousand languages and propose a new XML format for IGT. Methods for resource comparison and evaluation are described in Rodriguez-

Nancy Ide

ide@cs.vassar.edu

Nicoletta Calzolari

glottolo@ilc.cnr.it

1 Vassar College, Poughkeepsie, NY, USA

2 ILC-CNR, Pisa, Italy 
Fuentes et al., who introduce and evaluate a new database specifically designed to benchmark spoken language recognition technology; Pécheux et al. study the impact of reordering schemes on the performance of statistical machine translation systems; and Mori and Neubig provide an in-depth analysis of effects of augmenting dictionaries and training data on language models for Japanese word segmentation and part-of-speech tagging.

The article entitled "Rediscovering $15+1$ Years of Discoveries in Language Resources and Evaluation" (Mariani et al.) deserves special attention, as it provides an in-depth analysis and summary of activity in the field over the past decade and a half, based on data gleaned from LREC proceedings as well as proceedings of several related conferences. The article overviews trends in topics within the field and identifies networks of members of the LRE community based on analyses of mutual authorship and citation. Overall, it provides a unique perspective on the recent rapid evolution of research and development activity surrounding language resources, as well as documenting the growth and composition of community of active participants in the field.

We take this opportunity to announce that from now on, papers submitted to $L R E$ will be encouraged to include a separate section in the bibliography entitled "Language Resource References", in which all resources cited are given full reference, including the name of project or persons responsible for development of the resource, the date of its publication, the name and version number of the resource, and its method of distribution, with a specification of licensing or other requirements and information pertinent to obtaining the resource. ${ }^{1}$ Explicit identification of language resources in the bibliography serves a dual purpose: it provides at a glance information about the availability of resources used or cited in a journal article, and it ensures that resource developers are appropriately recognized by means of formal citation. As such, these citations further legitimize resource development and evaluation as a valuable scholarly activity.

In closing, we would like to recognize the members of the community who have contributed to the journal by reviewing papers, as well as those who have submitted to the journal over the past two years and the members of our Editorial Board. Their contributions are essential to maintaining the high quality of materials published in $L R E$ and the vibrancy of the field it represents.

\footnotetext{
${ }^{1}$ Formatting guidelines for language resource references are available on the LRE website at http://www.springer.com/education+\%26+language/linguistics/journal/10579.
} 